Epidemiology and Infection

http://journals.cambridge.org/HYG

Additional services for Epidemiology and Infection:

Email alerts: Click here

Subscriptions: Click here

Commercial reprints: Click here

Terms of use : $\underline{\text { Click here }}$

\title{
Descriptive epidemiology of enteric disease in Chennai, India
}

\author{
R. D. CHOPRA and M. S. DWORKIN
}

Epidemiology and Infection / FirstView Article / November 2012, pp 1 - 5

DOI: 10.1017/S0950268812001409, Published online: 17 July 2012

Link to this article: http://journals.cambridge.org/abstract S0950268812001409

How to cite this article:

R. D. CHOPRA and M. S. DWORKIN Descriptive epidemiology of enteric disease in Chennai, India. Epidemiology and Infection, Available on CJO 2012 doi:10.1017/S0950268812001409

Request Permissions : $\underline{\text { Click here }}$ 


\title{
SHORT REPORT \\ Descriptive epidemiology of enteric disease in Chennai, India
}

\author{
R. D. CHOPRA* AND M. S. DWORKIN \\ Department of Epidemiology and Biostatistics, University of Illinois, Chicago, IL, USA
}

Received 20 November 2011; Final revision 31 May 2012; Accepted 6 June 2012

\section{SUMMARY}

There are very few data on the prevalence of coccidian enteric parasites among low-income adults hospitalized in Chennai, India. Stool samples from 200 patients were screened for selected parasites, enteric bacterial pathogens, and other protozoa over a 3-month period. The study identified 42 (21\%) Cryptosporidium, 36 (18\%) V. cholerae, 17 (9\%) Salmonellla, 12 (6\%) Isospora, six (3\%) helminths, five (3\%) Shigella, one (1\%) Cyclospora, one (1\%) other protozoan, and $0 \% \mathrm{~V}$. parahaemolyticus cases. Co-infection was present in 21 patients. Cryptosporidium was detected in $17(81 \%)$ of co-infected patients. Our findings highlighted the relatively high proportion of patients in this population with Cryptosporidium and Isospora and suggest that further study be undertaken to determine the utility of broader use of diagnostic testing for coccidian parasites in India. Detection may be beneficial because isosporiasis is treatable and both Isospora and Cryptosporidium are important pathogens in AIDS patients.

Key words: AIDS, Cryptosporidium, Cyclospora, epidemiology, gastrointestinal infections.

In developing countries where clean and safe drinking water is not uniformly available, diarrhoeal disease is a common problem with a disproportionate number of persons affected coming from impoverished living conditions [1]. According to the WHO, an estimated 1 billion people lack access to improved water and 2.5 billion have no access to basic sanitation [1]. Cryptosporidium parvum is associated with $51 \%$ of water-associated outbreaks of parasitic protozoan diseases reported worldwide and Isospora belli with $1 \%$ [2]. We identified only one study in India with published information on environmental risk factors for adult infection with these organisms [3]. A recent review article on cryptosporidiosis also suggests that

\footnotetext{
* Author for correspondence: R. D. Chopra, Department of Epidemiology and Biostatistics, University of Illinois, $1603 \mathrm{~W}$. Taylor St, MC 92, Chicago, IL 60612, USA.

(Email: rchopr2@uic.edu)
}

the infection is a 'neglected disease' that typically affects the poorest populations and the authors call for a better understanding of the aetiology of diarrhoeal illness in this setting [2]. Cryptosporidium is also of interest because immunosuppressed individuals have increased morbidity and mortality when infected $[4,5]$ and the number of persons living with HIV in India was estimated as 2.4 million in 2009 [6].

The Communicable Diseases Hospital (CD Hospital) is the primary referral hospital for severe diarrhoeal disease in Chennai (Madras, population 6.5 million), India and is known to many as 'the cholera hospital'. Until 2009, routine testing at this hospital consisted of culture identification of Vibrio cholerae without diagnostic testing for other diarrhoeal agents. Shortly before our study began, Salmonella testing was added without speciation. We 
supplemented routine enteric microbiology laboratory diagnostic methods with the inclusion of coccidian parasite testing in order to describe the prevalence of these organisms in this population.

Epidemiological data from the CD Hospital was collected over a 3-month period to determine the prevalence of coccidian parasitism and identify environmentally related risk factors. Tamil- and English-speaking patients aged $\geqslant 18$ years who were admitted to the CD Hospital during July-October 2009 with diarrhoea were offered the opportunity to participate in this study by their attending physician. Only the first 5-10 patients admitted each day that agreed to participate were included, based on the limited availability of laboratory resources, until a sample of 200 patients was achieved. Individuals aged $<18$ years, and persons who could not speak either English or Tamil were excluded. Diarrhoea was defined as having $\geqslant 3$ watery stools per day.

A questionnaire administered by nursing staff in either English or Tamil, after patients had given their consent to participate, collected sociodemographic data and information on hygiene-related risk factors. All questionnaire and laboratory data were recorded in a Microsoft Access database and descriptive statistics were generated using SAS version 9.2 (SAS Institute, USA). However, because some patients declined to answer certain questions, descriptive statistics were calculated for some variables if the number was $<200$. For the purposes of hypothesis generation we examined environmental variables such as housing type and primary water source to determine if they might be associated with identification of coccidian parasites in stool samples. Stool samples were tested at the CD Hospital laboratory. Stools were enriched and then plated on triple sugar iron, SalmonellaShigella, Hektoen, and xylose lysine deoxycholate agar for identification of bacterial enteric pathogens (Salmonella, Shigella, V. cholerae, V. parahaemolyticus) by hydrogen sulfide, indole, citrate, urease, glucose, lactose, sucrose, maltose, mannitol, xylose, arabinose, Methyl Red, and Voges-Proskauer tests. Testing for Cryptosporidium, Cyclospora, Isospora, and other protozoa was established by means of modified Kinyoun acid-fast staining of faecal smears. Further speciation was not performed. Motility testing for helminths was also performed and reported as present or absent.

This study was reviewed and approved by the medical director of the CD Hospital and Chief Health Officer for the Corporation of Chennai in addition to the University of Illinois at Chicago Institutional Review Board.

From 25 July to 13 October 2009 we enrolled 200 adult patients from the CD Hospital. The median age ( $n=195)$ was 35 years (range $18-65$ years). The median monthly household income $(n=124)$ was 3000 rupees $(\sim £ 40)$ [range $1000-12000$ rupees $(\sim £ 13$ 163)]. For reference the 2004-2005 Tamil Nadu poverty line was 547 rupees $(\sim £ 7 \cdot 40)$ per capita monthly and the patients in this study had a median of three persons in their household with 23 patients falling below this level [7]. Therefore, we estimated that the household income of this patient population closely approximated the poverty line. The greatest proportion of patients in this sample had reported never attending school $(37 \%)$. Others reported attending primary school only $(31 \%)$, attending high school (24\%), and attending better than high school (9\%) $(n=196)$. Eighty percent of the patients came from Chennai and $20 \%$ came from a nearby semi-rural or suburban area outside Chennai called Moufussil. Type of housing $(n=199)$ was identified as pucca (houses made with permanent building materials, $48 \%$ ), kutcha (non-permanent houses made of makeshift materials, 20\%) and semi-kutcha (an intermediate between the two, $31 \%$ ). Immunosuppression was reported by $5 \%$ of patients after a brief explanation of common causes (e.g. AIDS, bone marrow transplantation, cancer chemotherapy, glucocorticoid therapy, leukaemia, lymphoma, multiple myeloma, malnutrition, or a primary immunodeficiency) by the nurse administering the survey $(n=189)$. Information on antibiotic usage prior to admission was difficult to interpret because it was reported by $110(55 \%)$ patients before admission yet only one patient was able to identify antibiotic treatment by name and other patients were only able to supply general terms in response to a request for which antibiotic they were given, e.g. 'antidiarrhoeal medicine' or that the patient had taken an 'unknown tablet'. Twenty-five received treatment at a hospital other than the CD Hospital and 85 reported over-thecounter or unspecified drug usage.

Of the 200 patients with stool specimens tested, we identified 120 pathogenic organisms from 92 patient stool samples (Table 1). The most common organism was Cryptosporidium (21\%) followed by $V$. cholerae $(18 \%)$. The median length of hospital stay was 2 days (range 1-7 days). Co-infection was identified in 21 patients (Table 2). Of the 21 persons with coinfection, Cryptosporidium was identified in $17(81 \%)$. 
Table 1. Identification of pathogenic organisms in stool samples from adults at the Communicable Diseases Hospital in Chennai, India $(N=200)$

\begin{tabular}{lrr}
\hline \hline Organism & No. positive & $\%$ \\
\hline Cryptosporidium & 42 & 21 \\
Cyclospora & 1 & 1 \\
Helminths & 6 & 3 \\
Isospora & 12 & 6 \\
Other protozoa & 1 & 1 \\
Salmonella & 17 & 9 \\
Shigella & 5 & 3 \\
V. cholerae & 36 & 18 \\
V. parahaemolyticus & 0 & 0 \\
\hline \hline
\end{tabular}

Data includes patients with co-infection.

Table 2. Identification of co-infection in stool samples from adults at the Communicable Diseases Hospital in Chennai, India $(N=200)$

\begin{tabular}{|c|c|c|}
\hline Organisms & $\begin{array}{l}\text { No. } \\
\text { positive }\end{array}$ & $\%$ \\
\hline Cryptosporidium, Isospora & 7 & 4 \\
\hline V. cholerae, Salmonella & 4 & 2 \\
\hline Salmonella, Cryptosporidium & 2 & 1 \\
\hline V. cholerae, Cryptosporidium & 2 & 1 \\
\hline Shigella, Cryptosporidium & 1 & 1 \\
\hline Cryptosporidium, V. cholerae and Isospora & 1 & 1 \\
\hline Cryptosporidium, Isospora and helminths & 1 & 1 \\
\hline $\begin{array}{l}\text { Cryptosporidium, Cyclospora, } \\
\text { Isospora and helminths }\end{array}$ & 1 & 1 \\
\hline Cryptosporidium and helminths & 1 & 1 \\
\hline Cryptosporidium and other protozoa & 1 & 1 \\
\hline
\end{tabular}

In patients with Cryptosporidium co-infection concurrent isolated organisms consisted of Isospora, Salmonella, Shigella, V. cholerae, helminths and other protozoa.

Most patients $(74 \%, n=197)$ reported a primary water source that was piped into their yard or dwelling. The prevalence of Cryptosporidium in patients whose primary source of water was municipal was 33 $(79 \%)$ and for Isospora seven (58\%). Boiling water for purification $(n=197)$ was reported by $19 \%$ of patients and filtration by $25 \%$. Boiling water for purification and water filtration was reported by $18 \%$ and $30 \%$ of Moufussil residents $(n=40)$, respectively, and by $20 \%$ and $24 \%$ of Chennai residents $(n=160)$, respectively. We also attempted to ascertain specific filtration and water purification methods through an open-ended question, but received a poor overall response rate (only $5 / 40$ patients who filtered their water). However, of these respondents four indicated that cloth fabric (sufficient only for filtering large particulates) was used as a filter and one patient reported that boiling or filtration was only undertaken during the rainy season. Chlorination (not defined or confirmed) of their primary water source was reported by $19 \%$ of patients $(n=197)$. Toilet type was reported as private $(47 \%)$, shared $(43 \%)$ and open air $(10 \%)$ by study participants $(n=197)$. Hand washing after using the toilet was reported as always $(51 \%)$, sometimes $(16 \%)$, seldom $(1 \%)$, never $(31 \%)$ and refused to answer $(2 \%)(n=197)$. Borderline statistically significant associations of Cryptosporidium were observed with several environmental variables (Table 3). Analysis of water source, education, severity of diarrhoea and vomiting, and toilet type did not reveal any statistical association with the isolated organisms. However, this study was not powered to detect these differences.

After introducing testing for coccidian parasites at a referral hospital for severe diarrhoeal disease in Chennai, we demonstrated $21 \%$ Cryptosporidium, $6 \%$ Isospora, and $1 \%$ Cyclospora infection. To our knowledge, only two studies have been published on the prevalence of coccidian parasites in Chennai among adults from 2000 until present $[8,9]$. Both of these studies consisted of HIV-positive patients with diarrhoeal illness which are not directly comparable to the general adult population with diarrhoeal illness in our study. However, both studies reported lower rates of Cryptosporidium than is present in our study sample. Additionally, a 2007 study in Chennai to ascertain faecal coliforms and parasitic oocysts in the city's municipal water supply showed that drinking water collected from several of the city zones of Chennai was positive for Cryptosporidium oocysts [10]. The high usage $(74 \%)$ of the city water supply as a primary drinking source in our population combined with the recent detection of oocysts in the municipal water supply could potentially lead to high seroimmunity in this population [11]. We are not aware of any current municipal water treatment in Chennai directed at Cryptosporidium. Due to these factors, we suspect that acute cryptosporidiosis should be less common than we observed in adults. This suggests that further study should be directed at determining if patient characteristics, such as immunosuppression from HIV infection, are responsible for this elevated prevalence.

In our general study population of adults, three markers of higher socioeconomic status (having a pucca compared to a kutcha or semi-kutcha home, and having a separate kitchen within the home, and 
Table 3. Selected associations of environmental and Cryptosporidium findings

\begin{tabular}{llll}
\hline \hline & \multicolumn{2}{l}{ Cryptosporidium } & \\
\cline { 2 - 3 } Characteristic & Yes & No & OR (95\% CI) \\
\hline Always or frequent hand washing after using toilet & $33(16 \cdot 8 \%)$ & $99(50 \cdot 5 \%)$ & $2 \cdot 71(1 \cdot 08-7 \cdot 71)$ \\
Seldom or never washing hands after using toilet & $7(3 \cdot 6 \%)$ & $57(29 \cdot 1 \%)$ & $1 \cdot 97(0 \cdot 93-4 \cdot 24)$ \\
Pucca-style home & $26(13 \cdot 1 \%)$ & $71(35 \cdot 6 \%)$ & $2 \cdot 27(1 \cdot 00-5 \cdot 05)$ \\
Semi-pucca or kutcha-style home & $16(8 \%)$ & $86(43 \cdot 2 \%)$ & $7(4 \%)$ \\
Having a separate kitchen & $15(8 \cdot 5 \%)$ & $127(72 \cdot 2 \%)$ & \\
Lacking a separate home kitchen & $27(15 \cdot 3 \%)$ & & \\
\hline \hline
\end{tabular}

OR, Odds ratio; CI, confidence interval.

increased hand washing after using the toilet) were borderline statistically associated with Cryptosporidium parasitism as was increased hand washing after using the toilet (Table 3). These associations are unexpected, but should be interpreted cautiously as this study was not powered to detect these differences; however, they allow for hypothesis generation. We suspect that prevalence might be associated with decreased exposure in this patient population and thus less immunity to Cryptosporidium over their lifetime in relation to their relatively higher socioeconomic status.

A limitation of these data may include an underestimation of diarrhoeal organisms due to treatment with antibiotics before admission to the CD Hospital. Further, due to cost limitations, Giardia and Campylobacter testing was not performed although these pathogens may be important in this region as well. Another limitation was the lack of testing for HIV. While our survey had a self-reported measure asking patients if they had immunosuppression due to a variety of listed medical conditions, this statistic is non-specific and could be influenced by the stigma of revealing HIV status or a lack of a recent or remote HIV testing. The severity of diarrhoeal illness of patients reported to the CD Hospital could also introduce a referral bias due to the heightened severity of enteric illness within this patient population.

These data suggest that coccidian parasites, especially Cryptosporidium and Isospora, are not rare in patients admitted with hospitalizable diarrhoea in Chennai. While there is no effective treatment for cryptosporidiosis, isosporiasis can be treated effectively with trimethoprim-sulfamethoxazole, a relatively inexpensive antibiotic. In addition, infection with Cryptosporidium or Isospora could indicate HIV infection, and therefore HIV testing of patients whose stool is positive for these coccidia could alert healthcare providers to undiagnosed persons. To determine if this testing is appropriate, additional studies are necessary to ascertain the degree to which adult acute cryptosporidiosis is associated with HIV-related immunosuppression in this under-served population. A study monitoring the prevalence of this parasite in the municipal water supply in order to determine if there are any predictable patterns may be useful in guiding announcements regarding when boiling or filtering water with appropriate filters (absolute pore size of $\leqslant 1 \mu \mathrm{m}$ ) may be most useful [12]. Such a study should include a full year of data collection to allow for consideration of possible seasonality of these parasites. These data also demonstrate the importance of performing descriptive epidemiological studies in developing world settings because they reveal that certain pathogens may be relatively prevalent. In the case of these coccidian parasites, their prevalence was higher than that of cholera at the time of the study and this was unexpected and enlightening for medical staff responsible for patient evaluation and treatment.

\section{ACKNOWLEDGEMENTS}

We thank Mr Theodore James (Communicable Diseases Hospital, Chennai) for overseeing laboratory testing during this study and all other hospital staff who supported this work. Funding was provided by a seed grant from the University of Illinois, Chicago.

\section{DECLARATION OF INTEREST}

None.

\section{REFERENCES}

1. World Health Organization. Diarrhoea: why children are still dying and what can be done, 2009. 
2. Putignani L, Menichella D. Global distribution, public health, and clinical impact of the protozoan pathogen Cryptosporidium. Interdisciplinary Perspectives on Infectious Diseases 2010, pp. 39.

3. Dwivedi KK, et al. Enteric opportunistic parasites among HIV infected individuals: associated risk factors and immune status. Japanese Journal of Infectious Diseases 2007; 60: 76-81.

4. Davis J. The massive waterborne outbreak of Cryptosporidium infections, Milwaukee, Wisconsin, 1993. In: Dworkin MS, ed. Cases in Field Epidemiology: A Global Perspective. Sudbury, MA: Jones \& Bartlett Learning, 2011, pp. 121-44.

5. Yoder JS, Harral C, Beach MJ. Cryptosporidiosis surveillance - United States, 2006-2008. Morbidity and Mortality Weekly Reports (Surveillance Summaries) 2010; 59: 1-14.

6. Joint United Nations Programme on HIV/AIDS (UNAIDS). Global report: UNAIDS report on the global AIDS epidemic, 2010.
7. Government of India. Poverty estimates for 2004-2005. Press Information Bureau, New Delhi, 2007.

8. Vignesh R, et al. High proportion of isosporiasis among HIV-infected patients with diarrhea in southern India. American Journal of Tropical Medicine and Hygiene 2007; 77 : 823-824.

9. Kumar SS, Ananthan S, Lakshmi P. Intestinal parasitic infection in HIV infected patients with diarrhoea in Chennai. Indian Journal of Medical Microbiology 2002; 20: $88-91$.

10. Anbazhagi M, et al. Cryptosporidium oocysts in drinking water supply of Chennai City, Southern India. CLEAN - Soil, Air, Water 2007; 35: 167-171.

11. Casemore D. Towards a US national estimate of the risk of endemic waterborne disease - seroepidemiologic studies. Journal of Water Health 2006; 4 (Suppl. 2): 121-163.

12. Centers for Disease Control and Prevention. Cryptosporidosis - a guide to water filters. Atlanta: Centers for Disease Control and Prevention, 2010. 